35 | 2009

Repenser la communication dans les organisations publiques

\title{
La mise en scène des débats publics au cœur de la communication des organisations publiques
}

Hélène Romeyer

\section{(2) OpenEdition}

\section{Journals}

Édition électronique

URL : http://journals.openedition.org/communicationorganisation/708

DOI : 10.4000/communicationorganisation.708

ISSN : 1775-3546

Éditeur

Presses universitaires de Bordeaux

Édition imprimée

Date de publication : 1 juin 2009

Pagination : $48-59$

ISSN : 1168-5549

Référence électronique

Hélène Romeyer, «La mise en scène des débats publics au cœur de la communication des organisations publiques », Communication et organisation [En ligne], 35 | 2009, mis en ligne le 01 décembre 2012, consulté le 04 mai 2019. URL : http://journals.openedition.org/ communicationorganisation/708 ; DOI : 10.4000/communicationorganisation.708 
Dossier : Repenser la communication des organisations publiques

\begin{abstract}
Résumé
Ce texte entend questionner les mutations de la communication publique et notamment celle des collectivités territoriales à travers l'exemple des collectivités grenobloises. Plus précisément, il s'agit de s'interroger sur l'utilisation d'un dispositif de débat public autour du développement des nanotechnologies dans la région grenobloise. Nous entendons montrer comment il est de moins en moins question de faire connaître l'action publique à travers la communication territoriale mais plus en plus de faire passer l'action publique elle-même en usant de ressorts communicationnels et notamment des dispositifs dits participatifs.
\end{abstract}

\title{
Mots-clés
}

Acceptabilité, action publique, communication publique, débat public, événementiel, gouvernance.

\section{Abstract}

This text intends to question the mutations of public communication and notably that of regions with a measure of autonomy across the example of the community of agglomeration of Grenoble. It is a question of showing the tool how communication serves political acceptability in the case of the choice of development of nanotechnologies

\section{Key-Words}

Public communication, public factual debate, gouvernance.

Hélène Romeyer est Maître de Conférences à l'Université Rennes1 (IUT Lannion). Elle est membre du Crape (UMR CNRS 6051) et du Gresec (EA 608). C'est dans ce dernier que l'étude proposée ici a été réalisée. 


\section{La mise en scène des débats publics au cœur de la communication des organisations publiques \\ Hélène Romeyer}

helene.romeyer@u-grenoble3.fr

Si de nombreux auteurs ont montré comment le processus de territorialisation et la multiplication des acteurs ont bouleversé la gestion publique territoriale (Duran et Thoenig, 1996 ; Hassenteufel, 1998), les spécialistes de la communication ont mis en exergue la façon dont les collectivités territoriales ont développé des outils de communication basés notamment sur l'interactivité et les techniques d'information-communication (Pailliart, Bouquillion, 2006). Ce texte entend analyser l'utilisation d'un outil particulier de la communication publique contemporaine : les dispositifs dits participatifs. Plus précisément, à travers l'étude d'un cycle de débats publics à Grenoble sur les nanotechnologies ${ }^{56}$, il s'agit de s'interroger sur l'utilisation de dispositifs délibératifs comme outil de communication. L'intérêt ici est à la fois l'outil de communication, à savoir le débat public, l'objet du débat, les nanotechnologies c'est-à-dire les sciences et techniques, et, le niveau d'organisation publique concerné, les collectivités territoriales. Dès lors cette expérience apparaît comme une forme d'aboutissement de la "démocratie technique (Callon, Lascoumes, Barthe, 2001) mais elle révèle aussi l'apparition de nouvelles compétences des collectivités territoriales. Dans ce contexte, l'outil délibératif se révèle être une réponse à la fois à un déficit de communication publique, et un outil de communication scientifique et technique.

En effet, la rencontre entre les débats parfois vifs sur les questions scientifique et technique, et les conséquences de la décentralisation, permet à travers l'exemple grenoblois de comprendre comment ces

\footnotetext{
${ }^{56} \mathrm{Ce}$ texte est issu d'une étude collective menée par six membres du Gresec (EA608) sous la direction d'I. Pailliart, dans le cadre du cluster 14, programme de recherche de la région Rhône-Alpes portant sur les Enjeux et représentations de la science, de la technologie et de leurs usages. (cf. http://erstu.ens-lsh.fr/). Ce programme a donné lieu à un rapport de recherche: "Médiation et médiatisation des sciences et des techniques : l'exemple du secteur des NST à Grenoble », novembre 2008, 128p.
} 
procédures se sont imposées. Puis, nous nous attacherons à montrer que, paradoxalement, l'utilisation de ce dispositif novateur révèle des attitudes institutionnelles et un réflexe de défense identitaire. Si le dispositif fournit une réponse politique à l'activisme communicationnel de différentes formes d'opposition ${ }^{57}$, c'est aussi un outil de réassurance.

\section{L'imposition du débat public}

Dans le cadre de la décentralisation, depuis 1982, l'État s'est progressivement dessaisi de plusieurs compétences au profit des collectivités territoriales $^{58}$. Sans la tutelle de l'État, les collectivités locales se lancent dans des projets de grande envergure. L'interpénétration entre élus, entreprises et société locale est ainsi devenue beaucoup plus forte et, toutes les collectivités territoriales, seules ou ensemble, sont désormais source d'initiatives et de projets en matière d'action publique (Pailliart, 2006). Cependant, les lois de décentralisation successives n'ont pas réussi à définir toutes les compétences des différents niveaux de collectivités, objet de la mission d'information de $2008^{59}$. Entre complémentarité et concurrence, les collectivités et leur communication doivent trouver leur place.

La décentralisation a donc tout à la fois abouti à la création ou à la modification de territoires posant des problèmes d'identification, et à l'élargissement des compétences des collectivités révélant des problèmes nouveaux de légitimité et de compétences. Le tout dans un contexte de défiance des Français vis-à-vis de leurs élus et de l'action politique comme en témoigne la désaffection pour l'action politique et les taux d'abstention relevés à chaque élection. Dans ces territoires, la communication est investie à la fois de la mission de rétablir le lien civique avec les citoyens, et de travailler sur la proximité et l'identification. Il s'agit désormais d'envisager la communication territoriale " comme réponse à la complexité des problèmes sociaux» (Mériaux, 2005, p.30) ${ }^{60}$.

\footnotetext{
${ }^{57}$ Dans le cadre de ce projet, nous avons observé les réunions préalables aux débats, procédé à une analyse de contenu méthodique des échanges, puis mené 24 entretiens semi-directifs avec les différents acteurs du projet.

${ }^{58}$ Les lois successives du 2 mars 1982, du 6 février 1992, du 4 février 1995, du 25 juin 1999, du 12 juillet 1999, du 13 décembre 2000, du 27 février 2002 , et du 13 août 2004.

${ }^{59}$ Mission qui a remis son rapport au gouvernement en octobre 2008.

${ }^{60}$ Cité par Pailliart I., 2006, p. 117.
} 
Le contexte social à Grenoble en 2005-2006 se cristallise autour des manifestations remettant en cause la politique de développement et d'investissement sur les nanotechnologies. En effet, la région grenobloise, héritière d'une tradition liant fortement scientifiques, industriels et élus, a choisi de promouvoir ce secteur, qui assure aujourd'hui une large part de l'activité économique et scientifique de la région: environ 24000 emplois directs ou indirects ${ }^{61}$. Cette politique d'investissement et de développement provoque une opposition protéiforme : groupes constitués (Attac, Pièces et Main d'œuvre, etc.), mouvements écologistes, actions syndicales, revendications citoyennes, médias alternatifs, élus de l'opposition. Les contestations portent à la fois sur la lourdeur des investissements, la condamnation d'une logique purement scientifique et technique, la crainte des conséquences d'une mono activité, la perte de repères identitaires du territoire, l'expansion du tissu urbain sous couvert de développement de l'innovation à tous crins. Des interrogations donc sur le «monde incertain» et les risques liés au développement scientifique (Callon, Lascoumes, Barthe, 2001).

Pour un certain nombre d'élus, quelle que soit la forme de ces interpellations, il convient de trouver un moyen d'y répondre. Pour ce faire, une étude est commandée au sociologue Pierre-Benoit Joly aidé par un groupe de personnalités scientifiques (Claude Gilbert ou Michel Callon par exemple), ainsi que des représentants de la fondation Science Citoyenne. Le groupe reçoit pour mission de «faire des recommandations sur les façons d'associer le public grenoblois au choix scientifiques et techniques ». Ainsi, preuve de la difficulté des élus face aux questions scientifiques, l'ensemble des collectivités territoriales commanditent une étude dans l'objectif d'être conseillé sur le dispositif le plus adéquat pour répondre aux interpellations dont ils sont l'objet. Et le dispositif préconisé est un outil de communication : le débat. Il s'agit donc de faire entrer les sciences et techniques dans l'espace public à une échelle locale. Instance de médiation et de débat public, l'espace public est de plus en plus fragmenté. Cette tendance s'affirme de plus en plus et donne lieu à la formation d'espaces partiels qui s'avèrent essentiels au fonctionnement «sociétal»: sciences et technologies, action culturelle et organisation de la vie de loisirs, organisation de la ville et des quartiers, forums favorisés par les nouveaux outils de communication, communautés dites virtuelles,

${ }^{61}$ Source AEPI, « Microélectronique et nanotechnologies », rapport décembre 2006. 
observation critique des médias de masses par les « publics », etc. sont autant de lieux où se développent des débats et se forment des opinions (Pailliart, 2005). L'objectif est donc d'observer les modalités de formation d'un espace public sur des questions scientifiques et/ou techniques à travers un dispositif de débat. Mais il s'agit aussi de " comprendre les modes de fonctionnement de la démocratie locale dans l'agglomération grenobloise $"{ }^{62}$ précisent les sociologues. De cette proposition, naît le projet Nanoviv, financé par l'ensemble des collectivités territoriales et dont l'organisation est déléguée conjointement à une association parisienne et au Centre de Culture Scientifique Technique et Industrielle (CCSTI) de Grenoble.

Six débats publics se déroulent ainsi de septembre à décembre 2006 autour de la thématique "Nano-biotechnologies : pour quoi faire? Comment ?». Chaque séance s'appuyait sur le travail préparatoire d'un groupe de conseillers (17 universitaires) et la présence de grands témoins, Nayla Farouki et Gérard Toulouse ${ }^{63}$.

Nanoviv naît donc de la congruence de plusieurs facteurs: l'émergence de divers mouvements revendicatifs qui interrogent désormais le niveau local des pouvoirs publics ; la nécessité de répondre aux critiques sur l'action publique locale; les craintes face à l'incertitude des conséquences et applications des recherches sur les nanotechnologies ; et la volonté des citoyens de prendre part aux décisions et choix de société. Il s'agit donc à travers cet événement de lutter à la fois contre le fossé grandissant entre sciences et société, et celui, non moins important entre élus locaux et citoyens. Les collectivités territoriales replacent ainsi le débat sur un terrain politique et selon des modalités qu'elles pensent maîtriser. Ce faisant, le processus délibératif devient un outil de communication publique.

\section{L'artifice délibératif pour parer à la complexité des questions scientifiques}

La question de la démocratisation des choix scientifiques est d'ordinaire posée à l'échelle nationale. Nanoniv tente donc de créer un

62 Pierre-Benoit Joly, «Démocratie locale et maîtrise sociale des nanotechnologies. Les publics grenoblois peuvent-ils participer aux choix scientifiques et techniques? », Rapport de la Mission pour la Métro, 22 septembre 2005. Document disponible à http://www.lametro.net/fr/projet_agglo/NanoGrenoble_rapport_final_05_09_22.pdf

${ }^{63}$ Nayla Farouki, philosophe et historienne des sciences, ou Gérard Toulouse, physicien à l'Ecole Nationale Supérieure de Paris. 
espace de discussion local. L'étude d'expériences similaires entre agglomérations et société civile offrent un panel contrasté de situations: certaines intercommunalités adoptent un système de médiation classique; d'autres au contraire ont réduit le champ d'influence des corps constitués au profit de forums plus proches du monde politique, des collectifs d'usagers ou des sphères privées (Faure, 2008). De fait, l'engouement croissant pour les expérimentations participatives depuis la Loi Chevènement et les nouvelles formules de consultations publiques traduisent l'intrusion du délibératif dans le champ des politiques publiques.

Il s'agit à travers cette expérience de relier pêle-mêle des velléités de démocratie participative, de démocratisation des choix scientifiques, et d'évaluer les capacités démocratique et délibérative des collectivités locales. Trois ans après, il est difficile de trancher sur l'objectif réel de cette expérience. L'aspect novateur de l'échelon local ne cache pas l'absence de réelle association des citoyens : d'une part, les décisions au moment des débats sont déjà prises, et, les financements votés et alloués, d'autre part les modalités délibératives ne révèlent pas réellement de débat. Ainsi, l'analyse des temps de parole montre une sur-représentation des scientifiques. Ces derniers ont bénéficié en moyenne de $40 \%$ du temps de parole total lors de ces rencontres. A contrario, le public, les citoyens ont pu s'exprimer sur un peu moins de $14 \%$ du temps de parole global, soit moins que les organisateurs $(21 \%)$ et à peine plus que les hommes politiques $(13,5 \%)^{64}$. A noter que paradoxalement c'est sur la thématique la plus sociétale, «les risques » lors des 2 heures 20 du débat 3 que les experts ont eu le plus la parole (plus d'1 heure et demi en interventions cumulées) et que le différentiel avec le public est le plus grand (environ 20 minutes en interventions cumulées).

Nous voudrions ici travailler l'hypothèse selon laquelle Nanoviv s'est transformé en outil de communication pour des questions pour lesquelles les élus locaux sont désarmés. Ainsi, les élus, en donnant une large exposition aux scientifiques ( $40 \%$ du temps de parole) ont délégué à ces derniers la responsabilité d'apporter des réponses. En effet, y compris parmi les élus soutenant le développement des nanotechnologies, certains font ouvertement état de difficultés à

\footnotetext{
${ }^{64}$ Les six rencontres sont fait l'objet d'une analyse de contenu ciblant particulièrement les modalités délibératives et les temps de parole des hommes politiques, des scientifiques, des organisateurs, des grands témoins et du public.
} 
débattre de questions scientifiques et techniques. Il est vrai que les interpellations sur les notions de risques, les conséquences environnementales, ou des interrogations éthiques, abordent des enjeux nouveaux pour ces élus locaux. Ces derniers s'interrogent alors sur leur légitimité, n'ayant ni l'habitude, ni les savoir-faire pour juger des incidences scientifiques ou éthiques de ces projets ${ }^{65}$. Et effectivement, le déroulement des débats laisse apparaître des thématiques sociales et éthiques peu habituelles pour des élus : la transformation du vivant, la bioéthique, les incidences en matière de santé, de libertés collectives, etc. "Les retombées positives de leur présence semblent faibles alors que la prise de risques est relativement forte : risque d'être mis en défaut ou d'être mis en cause » (Pailliart \& al., 2008, p. 61). Et ce, d'autant plus que le dispositif leur a en quelque sorte échappé : les élus sont absents du groupe de conseillers préparant les réunions, ils ne bénéficient d'aucun espace délimité lors des rencontres puisqu'il n'y a ni scène ni estrade, leurs interventions sont cadrées par les animateurs, et enfin les thématiques abordées sont peu politiques. A l'inverse, les scientifiques représentent la majorité de l'audience de ces soirées, toutes disciplines confondues. Ils se retrouvent à la fois dans les experts, mais aussi dans le public où nombre d'universitaires grenoblois avaient pris place. Bénéficiant d'un large temps de parole, leurs interventions se font massivement sur le mode explicatif ( Je vais essayer de vous montrer (...)» ; «Pour bien comprendre (...)» ; «Mon travail consiste en $\left.(\ldots) »^{66}\right)$.

Dès lors, à l'objectif premier d'entendre et de répondre aux critiques, s'est ajouté un objectif de réassurance d'abord politique, puis progressivement scientifique. Les acteurs scientifiques grenoblois se sont en effet massivement emparés du dispositif.

Schématiquement, quatre attitudes peuvent être isolées par les acteurs de ces rencontres :

- Pour les collectivités territoriales, il s'agissait avant tout d'afficher une volonté délibérative incarnée dans un événement ;

- Pour certains élus, il s'agit de prolonger un rôle d'opposant. Ainsi, lors du quatrième débat, les échanges furent monopolisés un temps par un élu de l'opposition remettant en cause le bien-fondé des décisions

65 Entretien avec le vice-président du développement universitaire scientifique et social de la communauté d'agglomération, 5 juillet 2006.

${ }^{66}$ Extraits d'interventions de personnalités scientifiques durant les débats. 
politiques. Et ce même si la lettre de mission adressée à l'association Vivagora, organisatrice des soirées était explicite sur l'objectif: "préciser l'objet des questionnements (...) favoriser la constitution d'une communauté d'échanges avec des acteurs académiques, industriels, associatifs ou élus, (...) et, donner l'occasion à un large public de comprendre les développements techniques, de saisir leurs enjeux et exercer un esprit critique $"{ }^{67}$. Il ne s'agissait pas de remettre en cause ou de délibérer des choix politiques déjà entérinés.

- Pour d'autres élus, Nanoviv aura été un moyen de faire formuler des réponses par des experts ou des acteurs extérieurs au milieu local. "On ne peut pas demander à des élus de répondre à la question "qu'est-ce que la science? ${ }^{68}$ déclare le chargé de la recherche du Conseil Général. De fait, les sujets scientifiques présentent une telle complexité et l'interrogation éthique sur leurs conséquences dépassant largement le cadre local, plusieurs hommes politiques ont entrevu dans ces soirées la possibilité d'obtenir des réponses sur des questions de plus en plus complexes. "Les débats ont servi à apporter des réponses à la population mais aussi aux élus $»^{69}$.

- Pour les scientifiques, ce fut l'occasion de venir expliquer ce qu'ils faisaient concrètement. Leur crédibilité ayant été remise en cause par les diverses manifestations c'était l'occasion pour eux de s'exprimer afin de combattre leur mauvaise image. Ainsi, un ingénieur du CEA déclarait lors d'une des rencontres : "Je suis jeune ingénieur (...) et je suis troublé. Je ne me reconnais pas dans le portrait fait de moi par les manifestants (...), mais je ne me reconnais pas non plus dans le portrait fait par le journal de la communauté d'agglomération $\gg{ }^{70}$. Il s'agissait alors de (re)légitimer sa place dans la société grenobloise.

La mise en scène des débats Nanoviv a donc permis certes de légitimer des choix, a servi d'outil d'acceptabilité des décisions politiques sous couvert de participation, et à œuvrer aussi à une certaine réassurance des élus locaux en terme de compétences. Mais, le dispositif a aussi religitimé les scientifiques et resitué leur rôle

\footnotetext{
${ }^{67}$ Extraits de la lettre de mission envoyée à l'association Vivagora par la communauté d'agglomération. Document transmis par la déléguée générale de l'association.

${ }^{68}$ Entretien avec le chargé de la recherche du Conseil Général de l'Isère, 27 mai 2007.

${ }^{69}$ Entretien avec le chargé de la recherche du Conseil Général de l'Isère, 27 mai 2007.

${ }^{70}$ Intervention du public, débat $\mathrm{n}^{\circ} 4$
} 
social après plusieurs mois de remise en cause à travers des manifestations parfois violentes dans les rues de Grenoble. Ce que traduisent bien à la fois leur présence importante lors des ces rencontres, et leurs prises de parole fréquentes.

Le paradoxe de cette initiative forte des collectivités territoriales est d'en avoir confié l'organisation à une association parisienne, Vivagora $^{71}$. Fondée en octobre 2003, l'association met en œuvre des débats sur les enjeux sociaux des choix scientifiques et techniques ${ }^{72}$. Pour ce projet, Vivagora s'est appuyé sur un certain nombre d'acteurs locaux $^{73}$ dont le CCSTI de Grenoble, réunis au sein d'un comité de pilotage. Concrètement, leur rôle est d'encadrer le débat, de proposer des intervenants et de participer aux séances d'évaluation des débats qui jalonnent le cycle, etc. Toutefois, le dispositif Nanoviv a confisqué la phase de "délibération ». Ainsi, systématiquement les rencontres débutaient par une longue mise en perspective, un cadrage de la part de Vivagora et le CCSTI (intervention de 11 minutes en moyenne), puis le public était invité à réagir et poser des questions (durant moins de 6 minutes en moyenne), et, les organisateurs donnaient ensuite la parole aux experts, non pas pour essayer de formuler une réponse aux questions du public mais pour réagir au cadrage initial. Il y a donc systématiquement rupture de la situation dialogique. Ces organisateurs sont sortis très largement de leur rôle de distributeur et facilitateur de parole. Issue du monde scientifique, la responsable de l'association a parfois monopolisé la parole avec en moyenne $21 \%$ du temps de parole. En outre, l'extériorité de Vivagora n'est qu'apparente. D'une part, Dorothée Benoit Browaeys, déléguée générale de l'association est une ancienne scientifique et ancienne journaliste scientifique ; Bernadette Bensaude-Vincent (intervenante lors de Nanoviv) est viceprésidente de Vivagora. L'association est également très proche des différents réseaux de débat public, et très investie dans les mouvements de réflexion autour de l'éthique des sciences. Ainsi, Gérard Toulouse (grand témoin pour Nanoniv), ancien membre du comité éthique du CNRS et défenseur au niveau national de ce mouvement, est également membre de Vivagora.

\footnotetext{
${ }^{71}$ Le montant de la prestation confiée à Vivagora pour la mise en œuvre de ce cycle de conférences s'élève à $23500 €$.

${ }_{72}^{72}$ ar exemple, le cycle de débat Nanomonde à Paris.

${ }^{73}$ Constitué essentiellement d'universitaires dont certains avaient un mandat au sein d'une des collectivités territoriale concernée et donc partie prenante dans Nanoviv.
} 
L'acteur extérieur vient donc ici renforcer la clôture de cette communauté locale «d'initiés» sur les questions scientifiques et le déroulement même des débats place la parole scientifique au-dessus des autres, et se répondant à elle-même. Cette expérience de processus délibératif révèle donc le caractère balbutiant d'un espace public scientifique (Miège, 2005).

\section{Conclusion}

Née d'une volonté politique, Nanoviv a mêlé trois niveaux d'interpellations: les questions posées par les nanotechnologies auxquelles aucune réponse ne fut apportée, au regret de plusieurs participants : "Sur les nanotechnologies, je voulais comprendre de quoi il s'agit et je n'ai rien appris.(...) Il n'en a pas été vraiment question $^{74} \gg$; le deuxième ensemble d'interpellations concerne les rapports entre sciences, politiques et société. Ils furent véritablement au cœur des débats, chacun essayant de légitimer son rôle et circonscrire ses responsabilités ; enfin, un ensemble de demandes répétées sur la nécessité de consulter la société sur ces questions et critiquant au passage les modalités de déroulement de ces débats : « un certain nombre de réponses n'ont pas été apportées (...) il y a eu surtout des débats à l'intérieur du débat (...) ». «Sur la forme, pour qu'un débat soit cohérent et constructif, que ça donne envie aux citoyens de participer, ben vous devriez nous donner la parole et dire stop aux spécialistes ou élus (...) ${ }^{75}{ }^{\prime}$.

S'agit-il d'une expérience délibérative ? Plusieurs indices l'infirment : un débat très contrôlé en amont, une circulation de la parole difficile, une surreprésentation d'universitaires ou de scientifiques, l'absence volontaire des principaux opposants. Les politiques, comme les scientifiques ou les militants opposants sont restés dans des rôles institutionnels, sans se saisir véritablement du dispositif. Il devient donc évident que Nanoviv s'est éloigné de son objectif délibératif et apparaît comme un outil de communication usant de l'événementiel. Pour la plupart des participants, ces soirées étaient moins des débats que des scènes publiques où il fallait afficher sa capacité à accepter le dialogue dans une sorte de quête de réassurance de la compétence des élus locaux, y compris sur les questions scientifiques et techniques.

$\mathrm{Au}$ terme de cette étude, il apparaît que l'histoire des procédures de délibération mises en place dans les collectivités locales et leur

\footnotetext{
${ }^{74}$ Entretien avec un participant, 6 décembre 2006.

${ }^{75}$ Interventions de deux participants au débat $n^{\circ} 4$.
} 
évolution ont poussé la communication des organismes publics vers des dispositifs innovants. Il ressort également de cette expérience qu'une confusion est entretenue entre dispositif délibératif et démocratie participative, de même qu'entre démocratisation et démocratie. La complexité des enjeux de l'expérience grenobloise pourrait aussi amener à penser la communication des organisations publiques à un niveau d'interterritorialité (Vanier, 2008) et non dans un contexte de territorialisation.

\section{Bibliographie}

CALLON, Michel. LASCOUMES, Pierre. BARTHE, Yannick. Agir dans un monde incertain. Essai sur la démocratie représentative. Paris : Seuil, 2001, 358 p.

DURAN, Patrice, THOENIG, Jean-Claude. L'État et la gestion publique territoriale in Revue française de Science politique, 1996, vol.46, n 4 , p. $580-623$.

FAURE, Alain. Des Contrats de ville aux Contrats de cohésion sociale : quel mouvement?. Les Cahiers du DSU. Janvier 2008, ${ }^{\circ} 47$, pp. 48-49.

HASSENTEUFEL, Patrick. Think social, Act local, la territorialisation comme réponse à la «crise» de l'Étatprovidence?, in Politiques et management public, vol. $16, \mathrm{n}^{\circ} 3$, septembre 1998, p.1-11.

JOBERT, Bruno. «Rhétorique politique, controverses scientifiques et constructions de normes institutionnelles : esquisse d'un parcours de recherche " in Faure A .La construction du sens dans les politiques publiques. Paris : L'harmattan, 1995, p. 13-24.

LATOUR, Bruno. Politiques de la nature, Paris : la Découverte, 1999, $382 \mathrm{p}$.

LE BART, Christian. Les maires : sociologie d'un rôle. Paris : Presses universitaires du Septentrion, 2003, 222 p.

MERIAUX, Olivier. Le débordement territorial des politiques sectorielles in Faure A., DOUILLET A.C. L'action publique et la question territoriale. Grenoble : Presses universitaires de Grenoble, 2005, p. 30

MIEGE, Bernard. «L'espace public sociétal Ô combien problématique » in PAILLIART, Isabelle, La publicisation de la science. Grenoble: Presses universitaires de Grenoble, 2005, p.125140. 
La mise en scène des débats publics...

MULLER, Pierre. «L'analyse cognitive des politiques publiques : vers une sociologie politique de l'action publique ", in Revue de science politique, vol. 50, $\mathrm{n}^{\circ} 2,2000, \mathrm{p} .189-208$.

PAILLIART, Isabelle (coord.), BORDEAUX Marie-Christine. CABEDOCHE Bertrand. CAUNE Jean. MIEGE Bernard. ROMEYER Hélène. Médiations et médiatisations des sciences et des techniques : l'exemple du secteur des NST à Grenoble. Rapport de recherche, novembre 2008, 130 p. [Document non publié]

PAILLIART, Isabelle. Territoires, identités et communication in OLIVESI S. Sciences de l'information et de la communication. Grenoble : Presses universitaires de Grenoble, 2006, p.113-128

PAILLIART, Isabelle. BOUQUILLION Philippe. Le déploiement des Tic dans les territoires. Le rôle des collectivités territoriales. Grenoble : Presses universitaires de Grenoble, 2006, 119 p.

VANIER, Martin. Le pouvoir des territoires. Essai sur l'interterritorialité. Paris : Economica.Anthropos, 2008, 160 p. 